\title{
Nephrosis and disturbances of neuronal migration in male siblings - a new hereditary disorder?
}

\author{
L PALM, I HÄGERSTRAND, U KRISTOFFERSSON, G BLENNOW, A BRUN, \\ AND C JÖRGENSEN
}

Departments of Paediatrics, Pathology, Clinical Genetics, and Obstetrics, University Hospital, Lund, Sweden

SUMmary Two male siblings (a boy aged 2 years 10 months at death and a male fetus aborted in gestational week 22) showed similar brain and kidney malformations, comprising paraventricular heterotopias, central canal abnormalities (including hydrocephalus in the boy), and glomerular kidney disease with proteinuria. There were no known hereditary diseases in the families of the parents, and there was one healthy sibling of either sex. The malformations thus seem to be hereditary in an autosomal or possibly $\mathrm{X}$ linked recessive fashion.

Disturbances in the development of the central nervous system during early gestation occur as the result of many different noxious factors and events. Infections, intoxications, and acute haemodynamic changes during early gestation may be the cause of migration disturbances such as neuronal heterotopias, cortical dyslamination, and other defects. Heterotopias have also been described in connection with several childhood syndromes. ${ }^{1}$ There are hereditary disorders among these, including comprehensive malformation syndromes where renal disease also occurs. Congenital nephrosis has been described in cases of microcephaly ${ }^{23}$ and in one case of migration disturbance. ${ }^{4}$

We report the connection between congenital nephrosis, migration disturbances, and central canal abnormalities in a boy and an aborted male sibling fetus, possibly constituting a new autosomal or $\mathrm{X}$ linked recessive syndrome.

\section{Patients}

The two cases presented here were the results of the second and third pregnancy of healthy, nonconsanguinous parents. There were no known hereditary disorders in the family. The first child, a healthy daughter, was born in 1977, when the mother was aged 22 . Subsequently, in 1984, after an uneventful fourth pregnancy, during which $\alpha$ fetoprotein concentrations and ultrasonography findings were normal, the mother delivered a healthy son.

Case 1. This boy was born at term by a normal delivery in 1979. No attempt at prenatal diagnosis was made. Birth weight was $3200 \mathrm{~g}$, with Apgar scores of $10-10-10$. Because of a radiologically verified aqueduct stenosis a ventriculoperitoneal shunt operation was performed at the age of 3 weeks. Just before operation a urinary tract infection was diagnosed associated with pyuria and moderate albuminuria. An excretory urogram and voiding cystourethrogram yielded normal results. The albuminuria disappeared after the urinary tract infection had been treated.

At 5 months the boy had a serous meningoencephalitis and a focal epileptic seizure of 30-40 minutes' duration. There were no signs of infection of the shunt. A nephrotic syndrome developed with continuing haemoglobinuria and albuminuria and a slight arterial hypertension. The nephrosis was first treated with a period of lactose free diet, during which the albuminuria temporarily diminished. Thereafter prednisolone was given without success. A renal biopsy examination was performed. Light microscopy showed very slight glomerular changes of proliferative type, and electron microscopy showed mesangial proliferation and swelling of the epithelial podocytes. No immunological deposits were found at electron microscopy or by histochemical examination.

Because of further febrile convulsions of long duration prophylactic treatment with phenobarbitone was given. At 10 months there were repeated severe general tonic-clonic seizures and at the same time the arterial hypertension increased. Because of the suspicion of nephritis induced by shunt infection the shunt was replaced. This operation was compli- 
cated by staphylococcal meningitis and an intraabdominal abscess. After abdominal surgery and treatment with antibiotics the new ventriculoperitoneal shunt had to be exchanged for a ventriculoatrial shunt. During and after these procedures the nephrosis continued with increasing amounts of albuminuria. Arterial blood pressure responded well to treatment. The boy was mentally retarded and developed a moderate spastic tetraplegia.

Treatment with cloxacillin was started and continued for about one year. During this time the clinical picture was fairly stable, but at 2.5 years the nephrosis worsened again. Intensive treatment with intravenous high dose, broad range antibiotics was tried without effect. Pulse treatment with methylprednisolone followed by long term treatment with oral prednisolone and cyclophosphamide led to

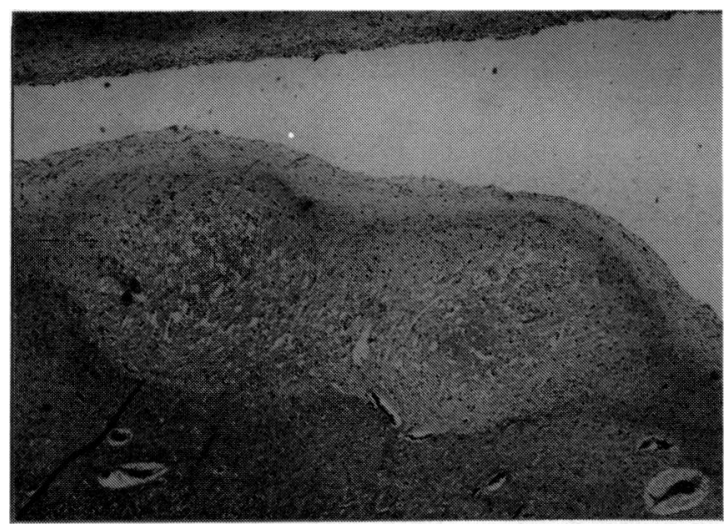

Fig. 1 A nodular heterotopia in one of the lateral ventricles from the child (haematoxylin-eosin stain).

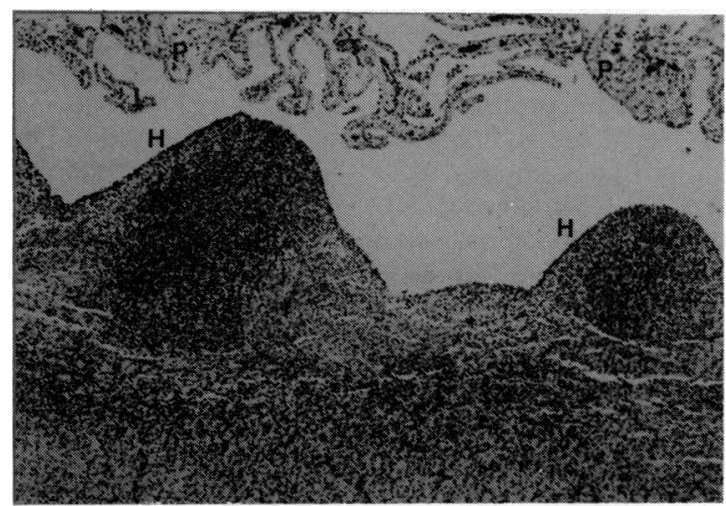

Fig. 2 Nodular heterotopias $(H)$ in one of the lateral ventricles from the fetus, with plexus choroideus $(P)$ in the upper part (haematoxylin-eosin stain). some improvement with rising serum albumin concentrations for a couple of months. At 2 years and 10 months the boy suddenly died during a respiratory tract infection.

\section{Autopsy}

The immediate cause of death was bronchitis. The brain weighed $1015 \mathrm{~g}$. The ventricular system above the sylvian aqueduct was dilatated. There were atrophic, thin gyri in the parieto-occipital region of one hemisphere, histologically with the occurrence of an old, large, incomplete cortical infarct. There were solid periventricular neuronal heterotopias bilaterally (Fig. 1).

The kidneys were of normal size and appearance. Together they weighed $136 \mathrm{~g}$. Many small pale dots were observed, both in the cortex and the medulla. They corresponded histologically to heaps of foam cells lying between tubular structures. The glomeruli showed no or very slight proliferative changes, and no progress of the glomerular lesions had occurred.

Case 2. This pregnancy was carefully followed. $\alpha$ Fetoprotein values of amniotic fluid in gestational week 17 were increased 20 -fold in two independent samples. The cytogenetic analysis was normal. Ultrasonography showed an oligohydramniosis, a broadening of the uppermost part of the vertebral column, and an increased ecogenicity from the kidney regions. Intrauterine growth was slowing

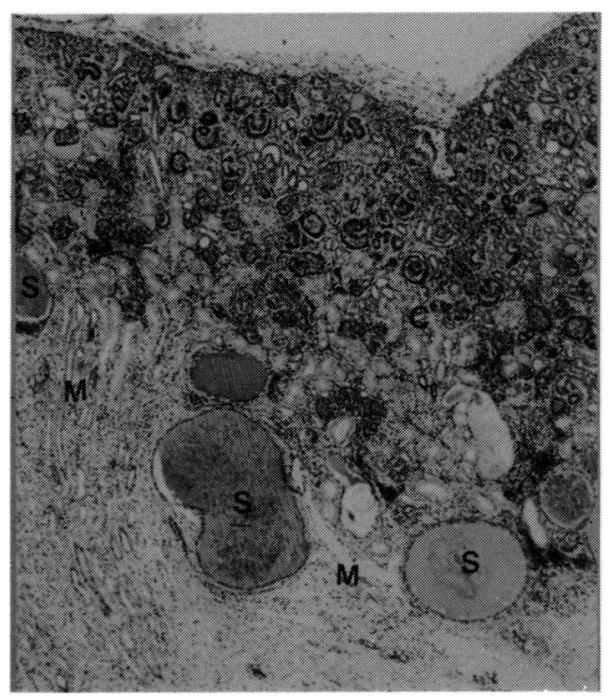

Fig. 3 The kidney from the fetus, showing many small cysts $(S)$ mainly on the border between cortex $(C)$ and medulla $(M)$ (haematoxylin-eosin stain). 


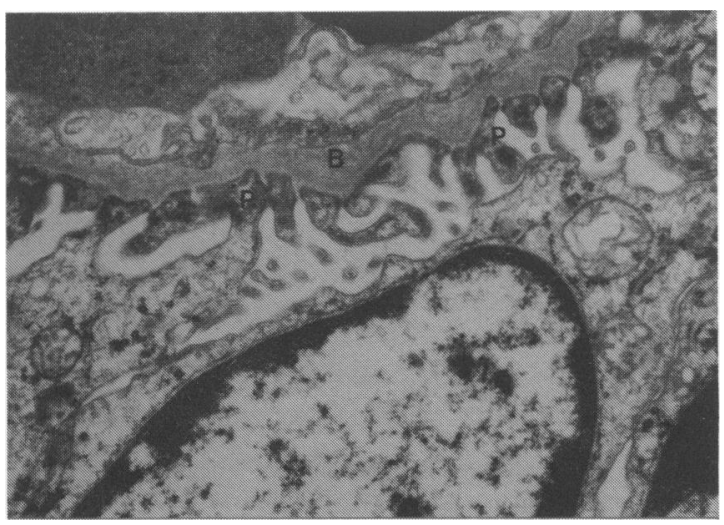

Fig. 4 Electron microscopy from the kidney of the fetus, showing normal podocytes $(P)$, but somewhat irregular basal membrane $(B)$ with varying density, and capillary lumen superior, epithelium inferior.

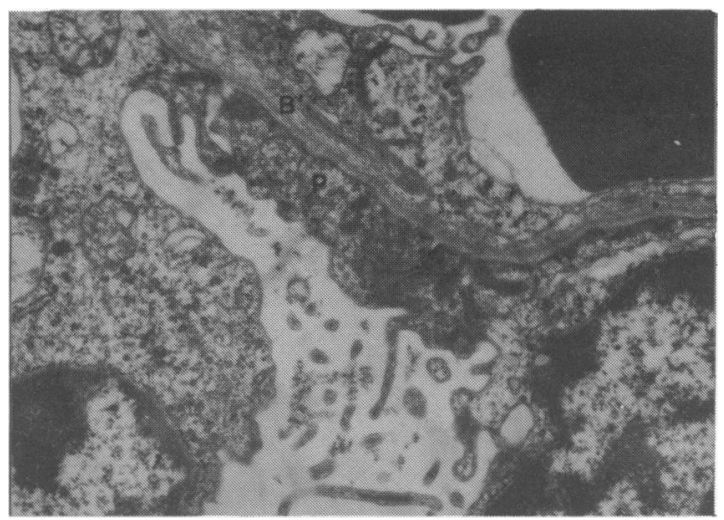

Fig. 5 Electron microscopy form the kidney of the fetus, showing abnormal, fused podocytes $(P)$, abnormal basal membrane $(B)$, and capillary lumen superior, epithelium inferior.

down. Legal abortion was made in gestational week 22.

\section{Autopsy}

The male fetus showed no gross malformations at autopsy. There was no hydrocephalus, and the kidneys were of normal appearance. Histologically, multiple neuronal, solid heterotopias were found in both lateral ventricles (Fig. 2). In sections from different parts of the spinal cord the central canal was found to be split into two or three tubes lying near each other or sometimes at considerable distance.

The kidneys showed histologically several small cysts lying mainly at the cortical-medullary junction. The cysts were lined by a rather high epithelium and contained eosinophilic fluid. Otherwise, the immature structures were judged to be normal. Electron microscopy disclosed slight glomerular changes, occasionally consisting of fused podocytes and irregularities of the basal membranes (Figs. 3-5).

\section{Discussion}

The main features of interest in this boy and his sibling fetus are the migration disturbance, the central canal malformation and the renal disease and the relation between them, as well as the possible hereditary nature of the disease.

If there is a common cause, genetic or environmental, for the renal and central nervous system malformation this must be searched for very early in gestation. The development of new kidney nephrons continues until gestational week 36 , and the earliest changes in congenital nephrosis might date back as far as the eighth to 10th week. In the fetal brain migration of neurons from the ventricular zone into the developing cortical plate takes place from the sixth to eighth gestational weeks onwards. The first cortical plate is formed in the eighth to 10th week. In the telencephalon the main process of migration is completed at about six months of gestation. ${ }^{5}$ In our study the fetus, 22 weeks old at abortion, was well past the most active migration period. The forking of the central canal in the fetus suggests a lesion about the time for closure of the neural tube early in neurogenesis. ${ }^{6}$ The development of the aqueductal stenosis shown on $x$ ray film in the elder brother is less clearly defined in time.

Several types of congenital hereditary nephrosis have been described, all with a suggested autosomal recessive inheritance. ${ }^{7}$ Congenital nephrosis of the Finnish type is well characterised as an autosomal recessive disorder. The kidney morphology in our boy, who died aged 2 years 10 months, however, does not agree with congenital nephrosis of the Finnish type, and children with this type of nephrosis do not usually survive infancy. In the fetus the presence of tubular cysts may fit in with the diagnosis of Finnish type nephrosis, although the glomerular findings are very discrete. ${ }^{8}$ Heterotopias have been described as the sole finding in monozygotic twins. ${ }^{9}$ No heredity was found in a series of five children with infantile spasms and heterotopias. ${ }^{1}$ Renal malformations and neuronal heterotopias have been described in syndromes with recessive heredity such as Zellweger's syndrome ${ }^{10} 11$ and Potter's syndrome. ${ }^{12}$ The combination of congenital nephrosis and migration disturbances has earlier been reported and considered important in one isolated case. ${ }^{4}$ Cases of congenital nephrosis together with microcephaly and hiatus hernia have 


\section{Palm, Hägerstrand, Kristoffersson, Blennow, Brun, and Jörgensen}

been reported in siblings but without description of disturbed neuronal migration. ${ }^{2} 3$

A distinct hereditary disorder may be considered in our cases. No other cases of nephrosis were known in this family, and there was no connection with Finland. Although the finding of two affected boys in the same family suggests an $\mathrm{X}$ linked inheritance of this new syndrome, an autosomal mode of inheritance cannot be excluded, especially as all previous reports of hereditary nephroses suggest an autosomal recessive mode of inheritance. ${ }^{7}$ In this context it should be mentioned that prenatal diagnosis in this disorder may be possible by $\alpha$ fetoprotein monitoring.

We are indebted to Dr Juhani Rapola, University Hospital, Helsinki, and Dr J R Pincott, the Hospital for Sick Children, Great Ormond Street, London, for their judgment on the kidney morphology.

\section{References}

' Palm L, Blennow G, Brun A. Infantile spasms and neuronal heterotopias. Acta Paediatr Scand 1986. (In press).

2 Galloway WH, Mowat AP. Congenital microcephaly with hiatus hernia and nephrotic syndrome in two sibs. J Med Genet 1968;5:319-21.

${ }^{3}$ Shapiro LR, Duncan PA, Farnsworth PB, Lefkowitz M. Congenital microcephaly, hiatus hernia and nephrotic syn- drome: an autosomal recessive syndrome. Birth Defects 1976;12(5):275-8.

${ }^{4}$ Robain O, Deonna T. Pachygyria and congenital nephrosis disorder of migration and neuronal orientation. Acta Neuropathol (Berl) 1983;60:137-41.

5 Rakic P. Neuronal migration and contact guidance in the primate telencephalon. Postgrad Med J 1978;54:25-40.

6 Källén B. Errors in the differentiation of the central nervous system. Birth Defects 1979;15(3):43-53.

${ }^{7}$ McKusick VA, ed. Mendelian inheritance in man. Catalogs of autosomal dominant, autosomal recessive and $\mathrm{X}$-linked phenotypes. 6th ed. Baltimore, United States: The John Hopkins University Press, 1983.

${ }^{8}$ Autio-Harmainen H, Rapola J. Renal pathology of fetuses with congenital nephrosis of the finnish type. Nephron 1981;29: 158-63.

9 Pavone L, Mollica F, Incorpora G, Pampiglione G. Infantile spasm syndrome in two monozygotic twins. Arch Dis Child 1980;55:870-2.

10 Della Giustina E, Goffinet AM, Landrieu P, Lyon G. A golgi study of the brain malformation in Zellweger's cerebro-hepatorenal disease. Acta Neuropathol (Berl) 1981;55:23-8.

1 Brun A, Gilboa M, Meuwisse GW, Nordgren H. The Zellweger syndrome: subcellular pathology, neuropathology and the demonstration of pneumocystis carinii pneumonitis in two siblings. Eur J Pediatr 1978;127:229-45.

12 Grunnet ML, Bale JF. Brain abnormalities in infants with Potter syndrome (oligohydramnios tetrad). Neurology (NY) 1981; 31:1571-4.

Correspondence to $\mathrm{Dr} \mathrm{L}$ Palm, Department of Paediatrics, University Hospital, S-221 85 Lund, Sweden.

Received 28 February 1986 